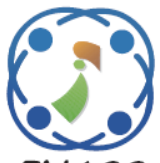

\title{
Multifocus Color Image Fusion Based on Walsh Hadamard Transform and Sum- Modified-Laplacian Focus Measure
}

\author{
Nainavarapu Radha ${ }^{1 *}$ \\ Tummala Ranga Babu ${ }^{2}$ \\ ${ }^{1}$ Aditya Engineering College, Surampalem, Andhra Pradesh, India \\ ${ }^{2} R V R$ \& JC College of Engineering, Guntur, A.P, India \\ * Corresponding author’s Email: radha_naina@yahoo.com
}

\begin{abstract}
Multi-focus image fusion objective is to add relevant information from multiple images of the same scene but with different focuses into a sharper image that is more suitable for visual sensor networks. Natural and artificially obtained multifocus color images are considered for fusion. The existing fusion methods like multi scale and multi-resolution transforms are proved to be good in Multi-focus Image Fusion. However, they suffer from computational complexity in kernels calculation. In this paper, Multi-focus color Image Fusion based on WalshHadamard Transform and sum-modified-Laplacian focus measure is proposed. Walsh-Hadamard Transform is a non-sinusoidal, orthogonal transform with symmetry, seperability and orthogonal properties. These properties make it more apt for image fusion than other transforms. And the sum-modified-Laplacian focus measure helps to get sharper image. Proposed method performance is evaluated in terms of reference and non-reference measures. The experimental results indicate that proposed method not only produces sharp details in fused image but also reduces the computational complexity.
\end{abstract}

Keywords: Walsh Hadamard transform, Multi-focus color image fusion, Sum-modified-Laplacian focus measure.

\section{Introduction}

In visual sensor networks, it became difficult to derive an image with all important objects in focus due to the restricted depth of focus of optical lenses in charge coupled devices (CCD) imagers. The solution to this is multi-focus image fusion, which adds multiple images of diverse focusing levels of the same scene into a sharper image which is more apt for visualization and detection. Multifocus image fusion has several applications in the areas of electronic circuit design and inspection, defence, computer vision, visual sensor networks and surveillance.

The multi-focus image fusion algorithms are classified into spatial and transform domain fusion methods. The spatial domain techniques $[1,2]$ use local spatial features such as gradient, spatial frequency, and standard derivation to fuse source images. However, the spatial domain techniques introduce undesirable effects such as image blurring and contrast reduction. For the transform domain methods, source images are projected onto localized bases which are generally made to denote the sharpness and edges of an image. In literature, various transform domain techniques involving multi scale decomposition were developed like Laplacian pyramid [3], Contrast and Gradient pyramids [4 - 6], principle component analysis (PCA) [7], multi-resolution transform methods such as multi-resolution singular value decomposition (MSVD) [8], discrete wavelet transform (DWT) [9], stationary wavelet transform (SWT) [10], lifting stationary wavelet transform (LSWT) [11], Daubechies complex wavelet transform (DCWT) [12], Shearlet Transform (ST) [13], double density discrete wavelet transform (DDDWT) [14] and discrete cosine harmonic wavelet transform (DCHWT) [15]. The major problem with pyramid based methods in [3-6] is lack of spatial orientation selectivity, which results in blocking effect in the fused image. PCA based method in [7] acquaint 
with blurring effect and does not contain fixed set of kernels. To overcome the limitations of PCA methods, the MSVD has been presented by author in [8]. But, MSVD also results edge discontinuity. In [9] author has presented multifocus image fusion using DWT. However lack of shift-invariance in DWT leads to aliasing effect in fused image. In [10 14] the SWT, LSWT, DCWT, ST and DDDWT are presented by authors for eliminating dearth of shift invariance caused by DWT. However there is computational complexity in kernels calculation and does not retain edges well and provides loss of contrast in fused image. To reduce the computational complexity in kernels calculation in [10-14], DCHWT has been presented by author in [15], which is a combination of discrete cosine transform (DCT) and Haar wavelet transform However, it introduces blocking effect due to DCT.

In multi-resolution transforms [8 - 15], 2D kernels are built by tensor product of 1D kernel. This building gives poor results in taking the geometrical constructions of real images and show discontinuity in edges. These methods also suffer from computational complexity in its floating point kernels calculation. To overcome such problems, Walsh-Hadamard Transform (WHT) presented by author in [16] is considered for image fusion with reduced complexity as the processing involves only kernels calculation in integer form compared to floating point kernels in multi-resolution transforms. In addition, a good fusion method not only relies on the transform but also depends on how to select the focused coefficients in transform domain. Thus, Focus measures are essential to differentiate focused regions from defocused regions in Multifocus image fusion to get sharper fused image. Several focus measures like contrast [17], variance [18], spatial frequency [19], and Sum-Modified-Laplacian [20] have been proposed in the literature for multifocus image fusion. The Sum-Modified-Laplacian (SML) has proven to be an effective focus measure by Huang in [20] for Multifocus image fusion. Hence, Walsh-Hadamard Transform in combination with SML focus measure is considered for image fusion in this manuscript.

\section{Preliminaries}

\subsection{Walsh Hadamard transform (WHT)}

Walsh-Hadamard Transform is a non-sinusoidal and orthogonal transform, decomposing an image into a function set which are Walsh kernels with values \pm 1 .
The 2-D WHT of an $\mathrm{N} \times \mathrm{N}$ image $f(x, y)$ is defined as given in Eq. (1):

$$
W H(u, v)=\sum_{x=0}^{N-1} \sum_{y=0}^{N-1} f(x, y) g(x, y, u, v)
$$

Equally, the inverse WHT (IWHT) is defined as given in Eq. (2):

$$
f(x, y)=\sum_{u=0}^{N-1} \sum_{v=0}^{N-1} W H(u, v) h(x, y, u, v)
$$

The quantities $g(x, y, u, v)$ and $h(x, y, u, v)$ are 2-D forward and inverse kernels (or basis functions) of WHT represented as given in Eqs. (3) and (4):

$$
\begin{aligned}
& g(x, y, u, v)=\frac{1}{N}(-1)^{\sum_{i=0}^{n-1}\left[b_{i}(x) p_{i}(u)+b_{i}(y) p_{i}(v)\right]} \\
& h(x, y, u, v)=\frac{1}{N}(-1)^{\sum_{i=0}^{n-1}\left[b_{i}(x) p_{i}(u)+b_{i}(y) p_{i}(v)\right]}
\end{aligned}
$$

Where $N=2^{n}, b_{i}(u)$ is the $i^{\text {th }}$ bit in the binary depiction of $u$. The quantities $p_{i}(u)$ and $p_{i}(v)$ are defined in terms of the $b_{i}(u)$ and $b_{i}(v)$ by Eqs. (5) and (6):

$$
\begin{gathered}
p_{0}(u)=b_{n-1}(u) \\
p_{1}(u)=b_{n-1}(u)+b_{n-2}(u) \\
p_{2}(u)=b_{n-2}(u)+b_{n-3}(u) \\
\cdot \\
\cdot \\
p_{n-1}(u)=b_{1}(u)+b_{0}(u) \\
p_{0}(v)=b_{n-1}(v) \\
p_{1}(v)=b_{n-1}(v)+b_{n-2}(v) \\
p_{2}(v)=b_{n-2}(v)+b_{n-3}(v) \\
\cdot \\
\cdot \\
p_{n-1}(v)=b_{1}(v)+b_{0}(v)
\end{gathered}
$$

As a result, the WHT contains in multiplying each image pixel by \pm 1 , summing, and dividing the sum by $\mathrm{N}$.

Properties of the WHT:

- The WHT is real, symmetric, and orthogonal, that is given in Eq. (7): 


$$
W H T=W H T^{*}=W H T^{T}=W H T^{-1}
$$

- Seperability: Seperability property is that $W H$ $(u, v)$ or $f(x, y)$ can be attained in two steps by consecutive application of 1-D WHT or its inverse. Hence, the 2-D WHT of $f(x, y)$ is defined using Eq. (8):

$$
W H T(u, v)=W H T^{y \rightarrow v}\left\{W H T^{x \rightarrow u}\{f(x, y)\}\right\}
$$

- The WHT is a fast transform. Since the WHT kernels are integers with values \pm 1 .

These properties make it apt for image fusion to produce a high quality fused image with reduced computational complexity.

\subsection{Sum-modified-Laplacian (SML)}

SML is a focus measure used to select focused areas from source images to build the fused image with every object in focus to achieve the objectives of multi-focus image fusion.

Nayar in [21], proposed a SML focus measure based on Laplacian of the image given in Eq. (9):

$$
\phi(u, v)=\sum_{(i, j) \epsilon(x, y)} \Delta_{m} I(i, j)
$$

Where $\Delta_{m} I$ the modified Laplacian of $I$, computed as (considering both neighbours in the $\mathrm{x}, \mathrm{y}$-directions) given in Eq. (10):

$$
\Delta_{m} I=\left|I * L_{X}\right|+\left|I * L_{Y}\right|
$$

The convolution masks used to compute the SML are defined in Eq. (11):

$$
L_{X=}\left[\begin{array}{lll}
-1 & 2 & -1
\end{array}\right], \quad L_{Y}=L_{X}^{T}
$$

Where $-L_{X}$ and $L_{Y}$ are convolution masks in $\mathrm{x}$ and $\mathrm{y}$ directions.

\section{Proposed image fusion algorithm}

The proposed algorithm is implemented in steps as follows:

Step1: Consider two source multi-focused color images (A \& B) for fusion.

Step 2: Transform source images in RGB space to YCbCr color space.

Step 3: The two source images were divided into equal blocks of size $n \times n$.

Step 4: Compute 1-level WHT for each block using Eq. (1) results $W H(u, v)$
Step 5: Calculate the SML focus measure of each block using Eq. (9-11).

Step 6: Select the corresponding focused block from each source images, according to the highest $\phi(u, v)$ using Eq. (12)

$$
W H_{F}(u, v)=\left\{\begin{array}{l}
W H_{1}(u, v), \text { if } \phi_{1}(u, v) \geq \phi_{2}(u, v) \\
W H_{2}(u, v), \text { otherwise }
\end{array}\right.
$$

Where $\phi_{1}(u, v)$ and $\phi_{2}(u, v)$ are the corresponding blocks with highest $\phi(u, v)$ from each source images. $W_{F}(u, v)$ is selected block from source images for fusion.

Step 7: The fused image is reconstructed by implementing IWHT using Eq. (2) on fused selected blocks in Step 6.

Step 8: inverse transform fused image in $\mathrm{YCbCr}$ to RGB color space.

Step 9: Calculate Reference measures of fused images in terms of Peak Signal to Noise Ratio, Structural Similarity Index Measure and Feature Similarity Index Measure using Eqs. (13) - (16).

Step 10: Calculate Non-reference measures of fused images in terms of Standard Deviation, Spatial Frequency and Mutual Information using Eqs. (17) (23).

\section{Performance evaluation measures}

The proposed method performance can be evaluated using reference and non-reference objective measures.

\subsection{Reference measures}

These measures evaluate performance of proposed method when the reference image is available.

\subsubsection{Peak signal to noise ratio (PSNR)}

It is an objective measure for fused image quality assessment. The reference image is given by $\mathrm{R}$ and fused image is represented by $\mathrm{F}$, Root Mean Square Error (RMSE) \& PSNR is calculated by Eq. (13-14):

$$
\begin{aligned}
& R M S E=\sqrt{\frac{\sum_{x=1}^{N} \sum_{y=1}^{N}[R(x, y)-F(x, y)]^{2}}{N \times N}} \\
& P S N R=10 \log _{10}\left[\frac{P_{\max } \times P_{\max }}{R M S E^{2}}\right]
\end{aligned}
$$




\subsubsection{Structural similarity index measure (SSIM)}

This measure quantifies fused image quality degradation. Representation of SSIM between the reference image $\mathrm{R}$ and fused image $\mathrm{F}$ is calculated as in Eq. (15):

$$
S S I M=\frac{\left(2 \mu_{R} \mu_{F}+C_{1}\right)\left(2 \sigma_{R F}+C_{2}\right)}{\left(\mu_{R}^{2}+\mu_{F}^{2}+C_{1}\right)\left(\sigma_{R}^{2}+\sigma_{F}^{2}+C_{2}\right)}
$$

\subsubsection{Feature similarity index measure (FSIM)}

It is an image quality metric that assesses the visual impact of two characteristics of a fused image: Phase congruency and gradient magnitude.

$$
F S I M=\frac{\sum_{x \in \Omega} S_{L}(x) P C_{m}(x)}{\sum_{x \in \Omega} P C_{m}(x)}
$$

Where $\Omega$ denotes the whole image in the spatial domain, $P C_{m}$ denotes phase congruent structure; $S_{L}$ is the similarity which depends on the gradient measure. The FSIM is defined using Eq. (16).

\subsection{Non-Reference measures}

These measures evaluate proposed method performance when the reference image is not available.

\subsubsection{Standard deviation (SD)}

It is used to evaluate the contrast of fused image. This is calculated as given in Eq. (17):

$$
\begin{gathered}
\sigma=\sqrt{\sum_{x=0}^{N} \sum_{y=0}^{N}(F(x, y)-\overline{\mathrm{F}})^{2}, \overline{\mathrm{F}}} \\
=\sum_{x=0}^{N} \sum_{y=0}^{N}|F(x, y)|
\end{gathered}
$$

\subsubsection{Spatial frequency (SF)}

To quantity the clarity level of fused image spatial frequency can be used. If the SF value is larger it denotes better fusion result and is calculated using Eqs. (18) - (20):

$$
R F=\sqrt{\frac{1}{N \times N} \sum_{x=1}^{N} \sum_{y=2}^{N}[F(x, y)-F(x, y-1)]^{2}}
$$

$$
\begin{gathered}
C F=\sqrt{\frac{1}{N \times N} \sum_{x=1}^{N} \sum_{y=2}^{N}[F(x, y)-F(x-1, y)]^{2}} \\
S F=\sqrt{R F^{2}+C F^{2}}
\end{gathered}
$$

\subsubsection{Mutual information (MI)}

Fused image quality is measured by mutual information. Large mutual information represents better quality. The MI between the source and fused images is given by Eqs. (21) - (23):

$$
\begin{aligned}
& I_{A F}(F, A)=\sum p(F, A) \log _{2}\left(\frac{p(F, A)}{p(F) \cdot p(A)}\right) \\
& I_{B F}(F, B)=\sum p(F, B) \log _{2}\left(\frac{p(F, B)}{p(F) \cdot p(B)}\right) \\
& S F=\sqrt{R F^{2}+C F^{2}}
\end{aligned}
$$

Where $p(F, A)$ and $p(F, B)$ - joint histograms of $(F$ and $\mathrm{A})$ and $(\mathrm{F}$ and $\mathrm{B})$

\section{Experimental Results and Discussion}

The proposed method has been implemented on artificially obtained multifocus color images and naturally obtained multifocus color images in $\mathrm{YCbCr}$ color space. The first experiment is performed using artificially created images with divergent focus levels. Airplane, standard image of USC-SIPI database [22] is considered as reference image. For each reference image, two artificially blurred images were produced by filtering the reference image with a $15 \times 15$ Gaussian filtered at the left and right parts respectively. Both the reference and artificially generated source images of Airplane are shown in Fig. 1.

Fused images from different fusion methods of Airplane are shown in Fig. 2. Demonstration of the proposed and different fusion techniques on artificially created images is presented in Fig. 2(a)(g). From Fig. 2 (a), one can find that DWT method [9] yields ringing artifacts in fused image due to lack of shift-invariance. The MSVD method [8] in Fig. 2 (b) shows discontinuities in edges. However, fused image of SWT method [10] in Fig. 2 (c) reduces the contrast in fused image. The DDDWT method [14] also yields blurring and ringing artifacts in fused image shown in Fig. 2 (d). The SF method [1] in Fig. 2 (e) introduces undesirable effects such as image blurring and contrast reduction. 


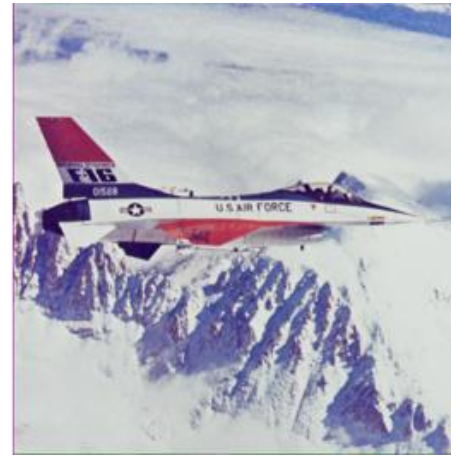

(a)

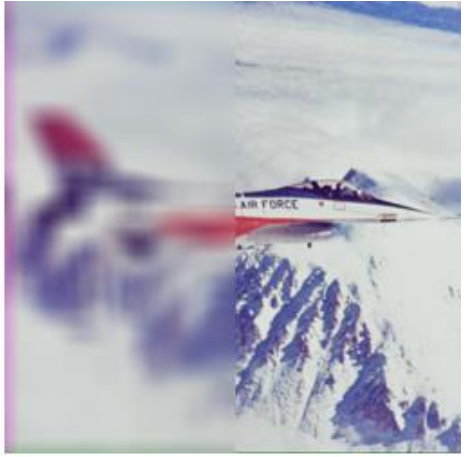

(b)

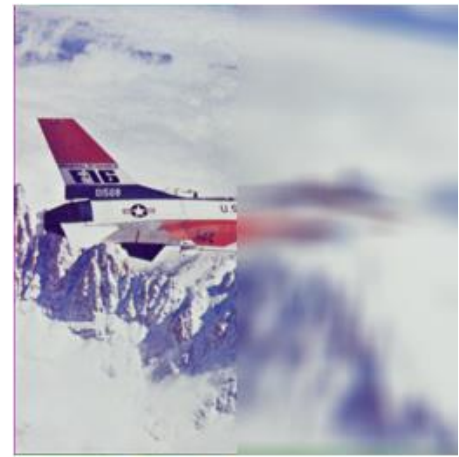

(c)

Figure. 1 Reference and source images of airplane: (a) reference image, (b) source image 1 (blur on Left), and (c) source image 2 (blur on Right)

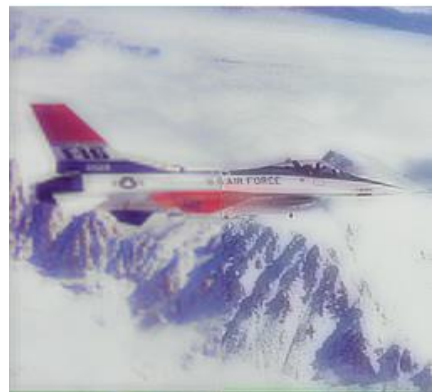

(a)

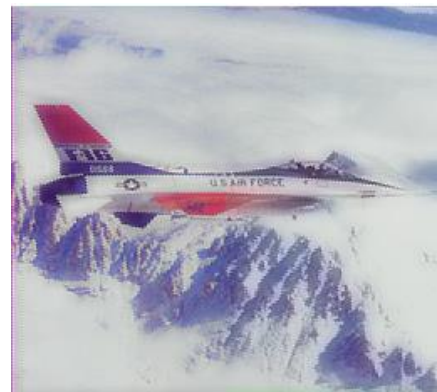

(b)

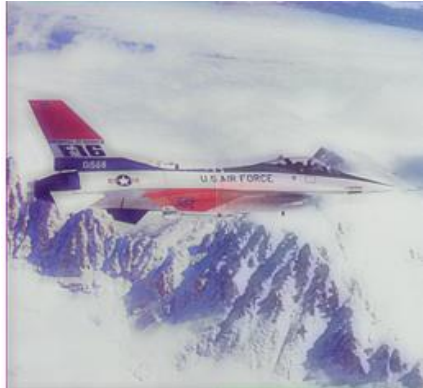

(c)

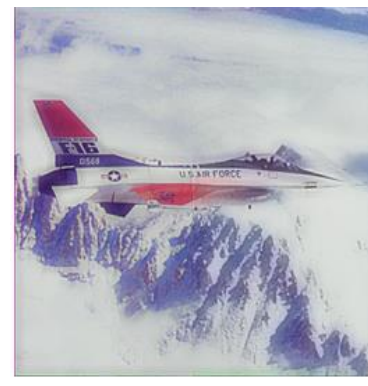

(d)

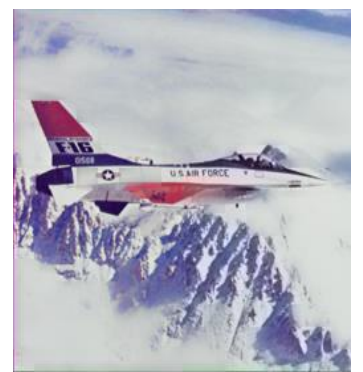

(e)

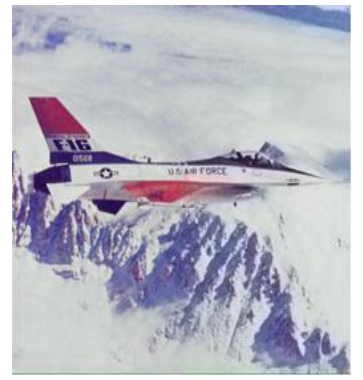

(f)

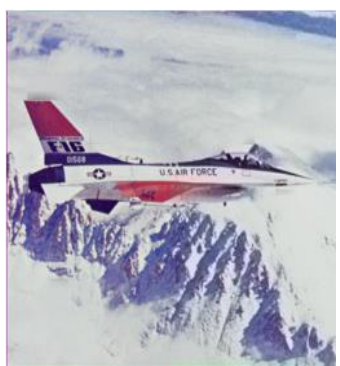

(g)

Figure. 2 Comparison of airplane fused images of different fusion methods (with reference image): (a) DWT [9], (b) MSVD [8], (c) SWT [10], (d) DDDWT [14], (e) SF [1], (f) DCHWT [15], and (g) proposed method

The DCHWT method [15] in Fig. 2 (f) shows blocking artifacts in fused image. One can observe from Fig. 2 (g) that the proposed method gives fused image without artifacts and good contrast. The logic is that applying the Walsh Hadamard Transform on each block of source images with simple kernel calculation provides shift-invariance which excludes artifacts and the use of Sum-modified-Laplacian focus measure selects focused regions from source images to get good contrast in fused image.

The performance of proposed method over different fusion techniques on artificially created images are described in terms of both reference and non-reference measures as given in Table 1. It can be observed from Table 1, that reference measures in terms of PSNR, SSIM, FSIM are high indicates that the fused image quality is good in proposed method. And non-reference measures in terms of MI, $\mathrm{SD}$, and SF of proposed method are also high shows that it preserves fused image details (focus regions) in a better way.

The second experiment is performed using naturally obtained multifocus color images with divergent focus levels. Man and map standard images of Lytro database [23] is considered for fusion. The source images of man are shown in Fig. 3. The experimental results are shown in Fig. 4. The fused image quality is defined in terms of improved contrast, sharp details like edges and boundaries. 
Table 1. Comparison of performance measures of proposed method with different fusion methods

\begin{tabular}{|c|c|c|c|c|c|c|}
\hline \multirow{3}{*}{ Fusion Method } & \multicolumn{6}{|c|}{ Airplane image } \\
\hline & \multicolumn{3}{|c|}{ Reference Measures } & \multicolumn{3}{|c|}{ Non-Reference Measures } \\
\hline & PSNR & SSIM & FSIM & MI & SD & SF \\
\hline DWT [9] & 25.3572 & 0.8147 & 0.8859 & 2.0822 & 36.5394 & 14.7071 \\
\hline MSVD [8] & 26.3356 & 0.8547 & 0.9173 & 3.7174 & 37.3520 & 19.3472 \\
\hline SWT [10] & 27.2888 & 0.8798 & 0.9253 & 3.8324 & 37.2858 & 17.5983 \\
\hline DDDWT [14] & 27.3447 & 0.9359 & 0.9238 & 3.7785 & 37.5176 & 19.0298 \\
\hline SF [1] & 31.3343 & 0.9540 & 0.9618 & 4.4343 & 42.4843 & 22.0440 \\
\hline DCHWT[15] & 33.0324 & 0.9691 & 0.9843 & 4.0134 & 42.2054 & 22.0469 \\
\hline Proposed & 33.1287 & 0.9721 & 0.9912 & 4.8161 & 43.4962 & 23.0732 \\
\hline
\end{tabular}

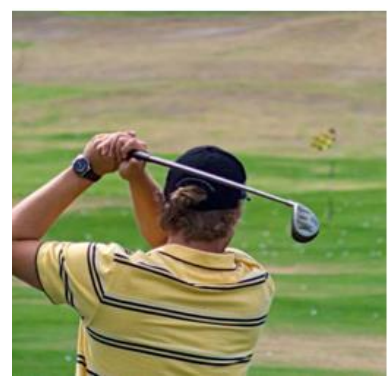

(a)

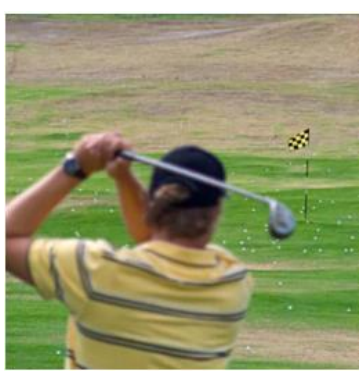

(b)

Figure. 3 Man source multifocus color images: (a) Source image 1(blur on background) and (b) Source image 2 (blur on foreground)

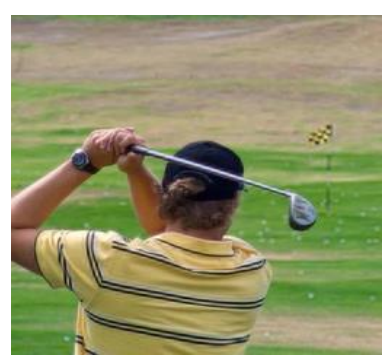

(a)

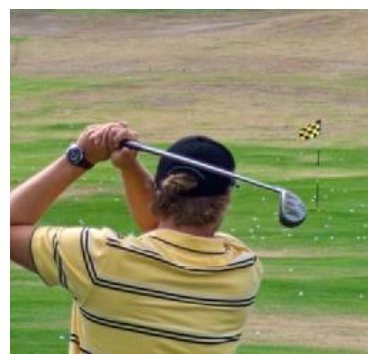

(b)

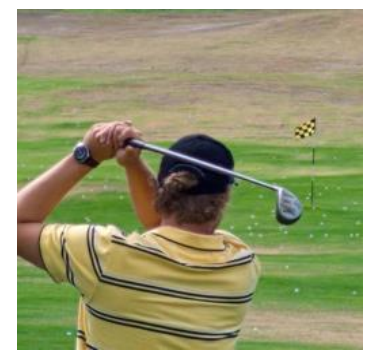

(c)

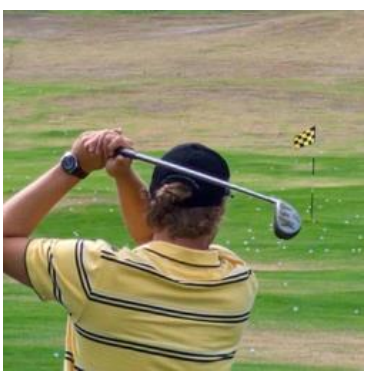

(d)

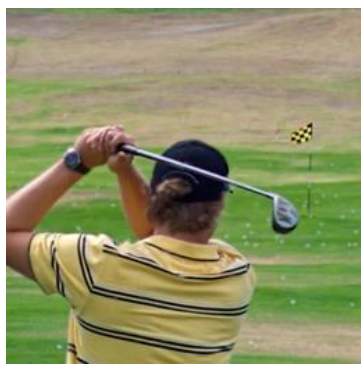

(e)

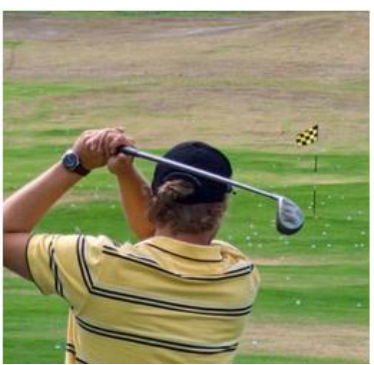

(f)

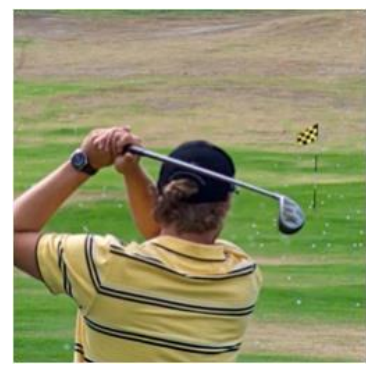

(g)

Figure. 4 Comparison of man fused images of different fusion methods (without reference image): (a) DWT [9], (b) MSVD [8], (c) SWT [10], (d) DDDWT [14], (e) SF [1], (f) DCHWT [15], and (g) proposed method

One can observe from Fig. 4(a)-(g) that the proposed method preserves edges and produce good contrast in fused image. From Fig. 4 (a), one can find that DWT method [9] yields blur on background in fused image. The MSVD method [8] in Fig. 4 (b) shows blur on foreground and discontinuities in edges.

However, SWT method [10] in Fig. 4 (c) reduces the contrast in entire fused image. The DDDWT method [14] also yields blurring in fused 
Table 2. Comparison of performance measures of proposed method with different fusion methods

\begin{tabular}{|c|c|c|c|}
\hline \multirow{2}{*}{ Fusion Method } & \multicolumn{3}{|c|}{ Man image } \\
\cline { 2 - 4 } & \multicolumn{3}{|c|}{ Non-Reference Measures } \\
\cline { 2 - 4 } & MI & SD & SF \\
\hline DWT [9] & 2.1128 & 44.4744 & 20.2360 \\
\hline MSVD [8] & 6.2384 & 44.8545 & 21.4021 \\
\hline SWT [10] & 6.5242 & 44.7263 & 20.5984 \\
\hline DDDWT [14] & 6.5699 & 45.0619 & 22.4433 \\
\hline SF [1] & 6.5768 & 45.5081 & 22.4940 \\
\hline DCHWT [15] & 6.4994 & 45.3556 & 22.7262 \\
\hline Proposed & $\mathbf{6 . 9 9 7 9}$ & $\mathbf{4 6 . 1 5 4 4}$ & $\mathbf{2 4 . 2 7 7 9}$ \\
\hline
\end{tabular}

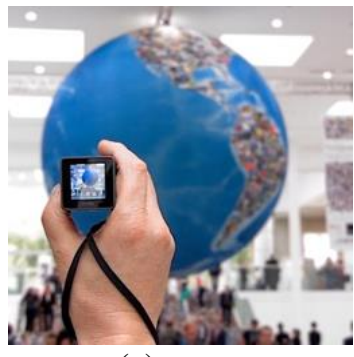

(a)

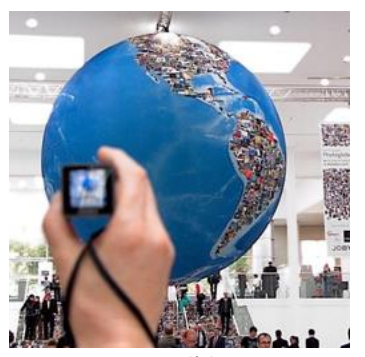

(b)

Figure. 5 The map non-reference source images: (a) foreground focused image and (b) background focused image

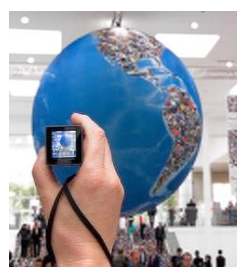

(a)

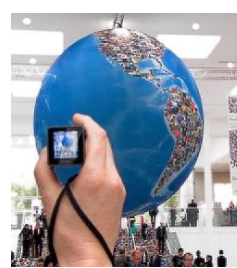

(b)

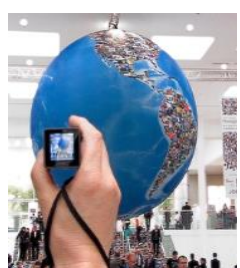

(c)

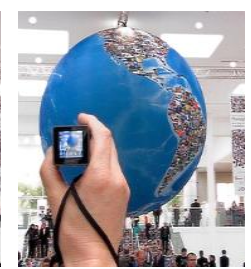

(d)

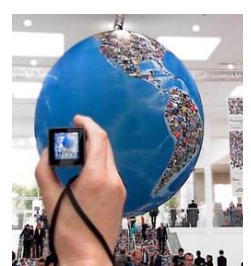

(e)

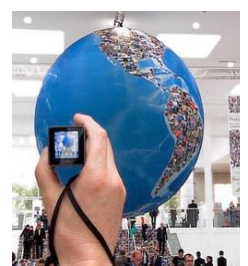

(f)

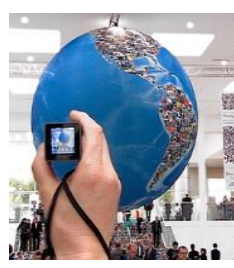

(g)

Figure. 6 Comparison of map fused images of different fusion methods (without reference image): (a) DWT [9], (b) MSVD [8], (c) SWT [10], (d) DDDWT [14], (e) SF [1], (f) DCHWT [15], and (g) proposed method

Table 3. Comparison of performance measures of proposed method with different fusion methods

\begin{tabular}{|c|c|c|c|}
\hline \multirow{2}{*}{ Fusion Method } & \multicolumn{3}{|c|}{ Map image } \\
\cline { 2 - 4 } & \multicolumn{3}{|c|}{ Non-Reference Measures } \\
\cline { 2 - 4 } & MI & SD & SF \\
\hline DWT [9] & 2.5392 & 70.0910 & 20.3644 \\
\hline MSVD [8] & 6.6877 & 71.7271 & 32.6054 \\
\hline SWT [10] & 6.9501 & 71.5852 & 31.3513 \\
\hline DDDWT [14] & 6.9519 & 71.9554 & 34.0584 \\
\hline SF [1] & 7.9401 & 73.1470 & 35.1219 \\
\hline DCHWT [15] & 7.1596 & 72.6975 & 35.2238 \\
\hline Proposed & $\mathbf{8 . 3 8 1 4}$ & $\mathbf{7 3 . 7 5 1 4}$ & $\mathbf{3 6 . 8 1 6 4}$ \\
\hline
\end{tabular}

image shown in Fig. 4 (d). The SF method [1] in Fig. 4 (e) introduces undesirable effects such as image blurring and contrast reduction. The DCHWT method [15] in Fig. 4 (f) shows blocking artifacts in fused image. One can observe from Fig. 4 (g) that the proposed method gives fused image with good contrast on both foreground and background. The performance of proposed method over different fusion techniques on naturally obtained images are described in terms of non-reference measures as given in Table 2. It is found from Table 2 that the proposed method has higher MI, SD and SF values compared to other fusion methods for all test images. High MI value indicates that the proposed method well transfers sharp details from source images to fused image. A high SD and SF value shows that contrast and edges are preserved in fused image. Similar observations are found in experimentation 
with map images as described in Fig. 5-6. The performance measures of proposed method are also high compared to other fusion methods as given in Table 3.

\section{Conclusions}

This paper presented a Multifocus color image fusion based on Walsh Hadamard Transform and Sum-modified-Laplacian focus measure. It not only preserved edges and focused details in fused image, but also solved the complexity of kernels calculation problem resulted in other fusion methods. In proposed method, Walsh Hadamard Transform is applied as its kernels calculation is simple, and Sum-modified-Laplacian focus measure is used for effective selection of focused regions from source images. The idea to get a sharper fused image with proposed method proved that it performs better than other fusion methods in terms of reference and nonreference performance measures. The proposed method is applicable for real-time applications in visual sensor networks and for fusion of microscopic images.

\section{References}

[1] S. Li, J. T. Kwok, and Y. Wang, "Combination of images with diverse focuses using the spatial frequency", Information fusion, Vol.2, No.3, pp.169-176, 2001.

[2] R. Vadhi, V. Kilari, and S. Samayamantula, "Uniform Based Approach for Image Fusion", In: Proc. of International Conf. On Eco-friendly Computing and Communication Systems, Springer, Berlin, Heidelberg, pp.186-194, 2012.

[3] R. Sabre and I. S Wahyuni, "Wavelet Decomposition in Laplacian Pyramid for Image Fusion", International Journal of Signal Processing Systems, Vol.4, No.1, pp.37-44, 2016.

[4] T. Pu and G. Ni, "Contrast-based image fusion using the discrete wavelet transform", Optical Engineering, Vol.39, No.8, pp.2075-2083, 2000.

[5] V. S. Petrovic and C. S. Xydeas, "Gradientbased multiresolution image fusion", IEEE Transactions on Image processing, Vol .13, No.2, pp.228-237, 2004.

[6] S. Paul, I. S. Sevcenco, and P. Agathoklis, "Multi-exposure and multi-focus image fusion in gradient domain", Journal of Circuits, Systems and Computers, Vol.25, No.10, p. $1650123,2016$.

[7] F. Sadjadi, "Comparative Image Fusion Analysis", In: Proc. of IEEE Computer Society
Conf. On Computer Vision and Pattern Recognition-Workshops, pp.8-8, 2005.

[8] V. P. S. Naidu, "Image fusion technique using multi-resolution singular value decomposition", Defence Science Journal, Vol.61, No.5, pp.479484, 2011.

[9] W. W. Wang, P. L. Shui, and G. X. Song, "Multifocus image fusion in wavelet domain", In: Proc. of International Conf. on Machine Learning and Cybernetics, pp.2887-2890, 2003.

[10] S. Li, B. Yang, and J. Hu, "Performance comparison of different multi-resolution transforms for image fusion", Information Fusion, Vol.12, No.2, pp.74-84, 2011.

[11] H. Li, S. Wei, and Y. Chai, "Multifocus image fusion scheme based on feature contrast in the lifting stationary wavelet domain", EURASIP Journal on Advances in Signal Processing, Vol.39, No.1, pp.1-16, 2012.

[12] R. Singh and A. Khare, "Fusion of multimodal medical images using Daubechies complex wavelet transform-A multiresolution approach", Information Fusion, Vol.19, pp.49-60, 2014.

[13] J. Liu, J. Yang, and B. Li, "Multi-focus Image Fusion by SML in the Shearlet Subbands", TELKOMNIKA Indonesian Journal of Electrical Engineering, Vol.12, No.1, pp.618 626, 2014.

[14] J. Pujar and R. R. Itkarkar, "Image Fusion Using Double Density Discrete Wavelet Transform", International Journal of Computer Science and Network, Vol.5, No.1, pp.6-10, 2016.

[15] B. S. Kumar, "Multifocus and multispectral image fusion based on pixel significance using discrete cosine harmonic wavelet transform", Signal, Image and Video Processing, Vol.7, No. 6, pp.1125-1143, 2013.

[16] S. Domnic, "Walsh-Hadamard transform kernel-based feature vector for shot boundary detection", IEEE Transactions on Image Processing, Vol.23, No.12, pp.5187-5197, 2014.

[17] G. Bhatnagar and B. Raman, "A New Image Fusion Technique Based on Directive Contrast", Electron Letters on Computer Vision and Image Analysis, Vol.8, No.2, pp.18-38, 2009.

[18] M. B. A. Haghighat, A. Aghagolzadeh, and H. Seyedarabi, "Multi-focus image fusion for visual sensor networks in DCT domain", Computers \& Electrical Engineering, Vol.37, No.5, pp.789-797, 2011.

[19] S. Li and B. Yang, "Multifocus image fusion using region segmentation and spatial frequency", Image and Vision Computing, Vol.26, No.7, pp.971-979, 2008. 
[20] W. Huang and Z. Jing, "Evaluation of focus measures in multi-focus image fusion", Pattern Recognition Letters, Vol.28, No.4, pp.493-500, 2007.

[21] S. K. Nayar and Y. Nakagawa "Shape from Focus", IEEE Transactions on Pattern Analysis and Machine Intelligence, Vol.16, No.8, pp.824-831, 1994.

[22] http://sipi.usc.edu/database/. (Accessed on 29/9/2017)

[23] http://mansournejati.ece.iut.ac.ir/content/lytromulti-focus-dataset. (Accessed on 29/9/2017) 